\title{
Bringing photosynthesis to the bench
}

\section{A series of experiments with genetically engineered chromatophores from purple bacteria suggests an unexpected coupling between molecular vibrations and the electron transfer at the heart of photosynthesis.}

Most of the understanding of the electronic properties of molecules accumulated in the past six decades rests on what is known as the Born-Oppenheimer approximation, the idea that the motions of atomic nuclei are so much slower than the characteristic times of electronic motions that the nuclei can be regarded as fixed in position while one calculates the electronic states. Quite apart from its plausibility in many circumstances, the assumption is also a great convenience: it allows the solution of problems that would otherwise be intractable. Quantum chemistry has flourished marvellously as a consequence.

But, as always, there must be exceptions. In the broadest sense, the problem tackled by Jean-Louis Martin and his colleagues at the INSERM Applied Optics Laboratory and described on page 320 of this issue is one of these. They are concerned with the process of charge transfer following the absorption of light quanta by the reaction centres of the photosynthesizing purple bacteria. The light-collecting elements consist of four molecules of bacteriochlorophyll, linked, together with quinone molecules, to electron acceptors known as bacteriopheophytins. There are specific protein appendages as well.

The reaction centres or chromatophores are embedded in membranes and, in the real-life photosynthetic process (as in that induced by the more familiar plant chlorophyll), the first step is the apparently irreversible transfer of electronic charge across the membrane. In bacteriorhodopsin, this process is known to take 3 picoseconds $\left(3.10^{-12}\right.$ seconds) at ordinary temperatures, perhaps only a quarter of that time at $10 \mathrm{~K}$.

What Martin and his colleagues have shown is that this reaction time is comparable with and even less than that for which the motion of the atomic nuclei of the protein parts of the reaction centre are coherent with each other. In other words, it may be inferred, there are molecular vibrations whose timescale is comparable with the time taken for the electron transfer process, and which therefore cannot be left out of account in discussions of the first step in bacterial photosynthesis.

This is a technical tour de force, combining esoteric techniques from laser spectroscopy with those from genetic engineering. Thus the study has been made possible only by the genetic engineering of one of the protein components of the reaction complex so that actual electron transfer does not take place; instead, on the absorption of a light- quantum, the complex remains in an excited state whose properties can be followed for several picoseconds.

The essential observation, that the fluorescence of this excited state seems to be periodic on a picosecond scale, was first published 18 months ago (Proc. natn Acad. Sci. U.S.A. 88, 8885-8889; 1991). One interpretation of that result was that the periodicity of the fluorescence is indeed a consequence of the coherent molecular vibration of one of the protein components of the photosynthetic reaction complex. The new development is that the interpretation has been shown to be correct.

That may sound simple enough, but it is not. For one thing, to study phenomena lasting for a few picoseconds, it is necessary to excite them by means of laser pulses whose duration is even shorter, lasting for just femtoseconds (where a femtosecond is $10^{-15}$ seconds). Similarly, the analysis of the fluorescence of the excited complex must be comparably sensitive in time (which is where the novelty of this account of the research resides). But verisimilitude also requires that the bacterial reaction complex should be embedded in real bacterial membranes, while the need that the signal should not be buried in the noise requires measurement at $10 \mathrm{~K}$. That could well have been a recipe for a small army of researchers, not just five of them.

What the authors do is to excite the bacterial reaction complexes with a femtosecond pulse of laser light centred on a near-infrared wavelength between 850 and $900 \mathrm{~nm}$. Because the duration of the pulses is a few tens of femtoseconds, the effective spread of wavelength (which is inversely proportional to the duration of the pulses) is always great enough to span the absorption band. Oscillations in the output are apparent to the eye in the fluorescent output. Fourier transforms of the output then yield the frequencies of the underlying vibrations which are supposedly their origin.

It is striking that the spectroscopy is evidently good enough for the authors to engage in discussions of their data comparable in detail (and complexity) with those familiar in the spectroscopy of simple diatomic molecules. The vibration that seems to matter most is that whose wavenumber (a proxy for frequency) is $70 \mathrm{~cm}^{-1}$. (There is another frequency at $15 \mathrm{~cm}^{-1}$ that also seems repeatedly to crop up.) That is well into the infrared, as the frequency of a molecular vibration should be.

The similarity between the time-span of the electron transfer process and the time- span of the vibrations of the protein molecules may be fortuitous, but that is to beg the question that interests others as well as Martin and his colleagues. Is it just an accident that the molecular vibration has just the frequency to confound the fluorescent output of the excited state that would normally result in electron transfer? Or is it possible that the molecular vibration is an essential part of the process, perhaps contributing to the very high efficiency of the electron transfer?

For what it is worth, the authors seem (appropriately judiciously) to be leaning towards the idea that the vibrations do indeed impel the process of electron transfer. Certainly the vibrations, which identify themselves by the relative persistence, appear to be unusual in their freedom from the dissipation they might be expected to suffer from. But it is far from clear just what vibration mode of a protein molecule, which has no direct part in the gathering of light, could play this trick.

Evidently it is within the bounds of possibility that these questions could be adjudicated by experiment. If it has been possible by genetic engineering to produce reaction complexes that do not allow electron transfer, why not alter the constitution of the protein molecules involved in such a way that their vibrational spectrum is changed and then see whether the efficiency of the transfer is reduced? That task seems well within the competence of Martin and his colleagues.

But what proteins to alter, and in what way? The trouble, as things stand, is that there is no way of calculating the vibrational frequencies of such a complicated structure as the reaction complex, especially within its natural environment of a membrane structure. Molecular dynamics or some variant of it seems the best way forward, but only with some difficulty.

Of course, on the assumption that the protein vibrations are indeed some kind of driving force for the electron transfer, it may be possible to guess at the symmetry or some other property of the vibration by estimating the transition probabilities between the excited state and that with a transferred electron, but that would require an element of luck. In the circumstances, it is commendable that Martin and his colleagues do not claim a big step forward in the understanding of photosynthesis. But it is an important step forward in that it could become the springboard for a big step. 